\title{
A Three-Dimensional Beam Profile Monitor based on Residual Gas Ionization
}

\author{
D. Shapira, T.A. Lewis \\ Physics Division, Oak Ridge National Laboratory, Oak Ridge. Tennessee 37831. USA
}

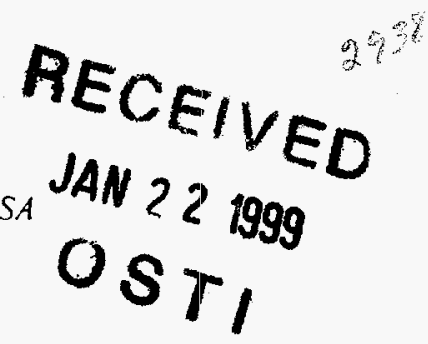

A three-dimensional beam profile monitor based on tracking the ionization of the residual gas molecules in the evacuated beam pipe is described. Tracking in position and lime of the ions and electrons produced in the ionization enables simultaneous position sampling in three dimensions. Special features which make it possible to sample very low beam currents were employed.

\section{INTRODUCTION}

Nonintrusive beam sampling has been used in storage rings and other facilities where high-quality low-emittance charged particle beams have to be stored for extended periods. The first devices which utilize residual gas ionization for beam sampling have been developed and applied extensively at such facilities. (1-7) In all the cases cited above the beam profiles were sampled only along one direction transverse to the beam. Two separate devices had to be used in order to sample two orthogonal projections transverse to the direction of propagation of the beam. Figures 1 and 2 show typical arrangements for these devices two years ago.

The residual gas molecules in the beam pipe are occasionally ionized and the positive ions are extracted with an electric field and impact on a position sensitive detector. This yields information on the beam location along one transverse dimension. When a full profile is desired two such devices were employed in tandem. This did not yield a true profile of the beam, but rather, only two distinct projections. The construction, instrumentation and testing of a true three dimensional beam profile sampler which can operate with very low beam intensities is described in this article.

\section{PRINCIPLES OF OPERATION}

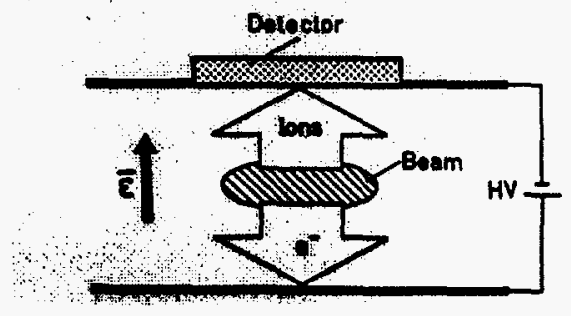

Fig. 1. Schematic diagram showing the principle of operation of the residual gas beam monitors.
The principle, illustrated in Fig. 3, is based on sweeping out the electrons and the positive ions produced in ionizing collisions between the beam particles and the residual gas molecules in the evacuated beam pipe. The beam particles have large kinetic energies and continue unabated but the residual ions drift in the direction of the electric field (toward the more negative electrode) and the knocked out electrons move in the opposite direction. The extraction electrodes are made with a $94 \%$ transmission grid. The charged ions and electrons pass through the grid and are then further accelerated and amplified with microchannel detectors (MCP).

Spatial resolution is limited in principle by transverse momentum transfer to the residual gas ion by the beam The lateral displacement is given by:

$\Delta r=\sqrt{\frac{2 M z}{q \Delta V / D}} v \perp=\sqrt{\frac{2 M c^{2} z}{q \Delta V / D}} \beta \perp$

where $M=$ mass of residual gas ion, $v \perp=$ transverse velocity of residual gas ion. $z=$ initial displacement from cathode, $\Delta V=$ applied voltage across detector and $D=$ distance between plates. Typical position resolution achievable with $H_{2}^{+}$ions is around $300 \mu \mathrm{m}$ (see Ref. 5).

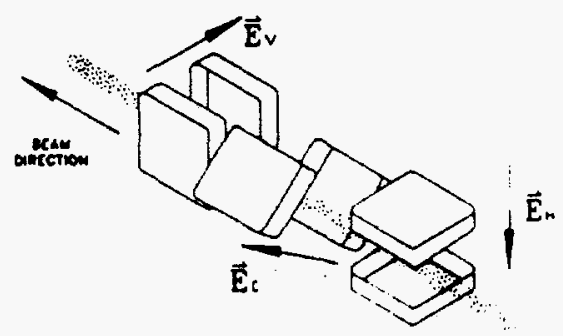

Fig. 2. Arrangment of detectors employed when a two dimensional profile of the beam was desired. 


\section{DISCLAIMER}

This report was prepared as an account of work sponsored by an agency of the United States Government. Neither the United States Government nor any agency thereof, nor any of their employees, make any warranty, express or implied, or assumes any legal liability or responsibility for the accuracy, completeness, or usefulness of any information, apparatus, product, or process disclosed, or represents that its use would not infringe privately owned rights. Reference herein to any specific commercial product, process, or service by trade name, trademark, manufacturer, or otherwise does not necessarily constitute or imply its endorsement, recommendation, or favoring by the United States Government or any agency thereof. The views and opinions of authors expressed herein do not necessarily state or reflect those of the United States Government or any agency thereof. 


\section{DISCLAIMER}

Portions of this document may be illegible in electronic image products. Images are produced from the best available original document. 
Table 1. Probability of ion-pair creation predictions for ionization by $6 \mathrm{MeV} \alpha$ particles in the residual gas.

\begin{tabular}{cccccc}
\hline Gas & $\mathrm{T}\left(\mathrm{ng} / \mathrm{cm}^{2}\right.$ & $\Delta(\mathrm{eV})$ & Ion Pot $(\mathrm{eV})$ & E+l Prod(eV) & No. of pairs \\
\hline $\mathrm{CO}_{2}$ & 56.8 & $42^{*} 10^{-3}$ & 15.0 & 200 & $2.1^{*} 10^{-4}$ \\
$\mathrm{~N}_{2}$ & 36.2 & $26^{*} 10^{-3}$ & 15.0 & 196 & $1.4^{*} 10^{-4}$ \\
$\mathrm{H}_{2}$ & 2.58 & $6.65^{*} 10^{-3}$ & 15.0 & 65 & $1.0^{*} 10^{-4}$ \\
\hline
\end{tabular}

In our version of this device we also detect the electrons formed in the same interaction. Because of their larger initial velocities, the electrons provide somewhat inferior position resolution, but they move much faster toward the electrode and their time of arrival is used to trigger a measurement of the drift time of the ions arriving to the opposite electrode. The difference in drift time for ions and electrons generated at the interaction point is given by

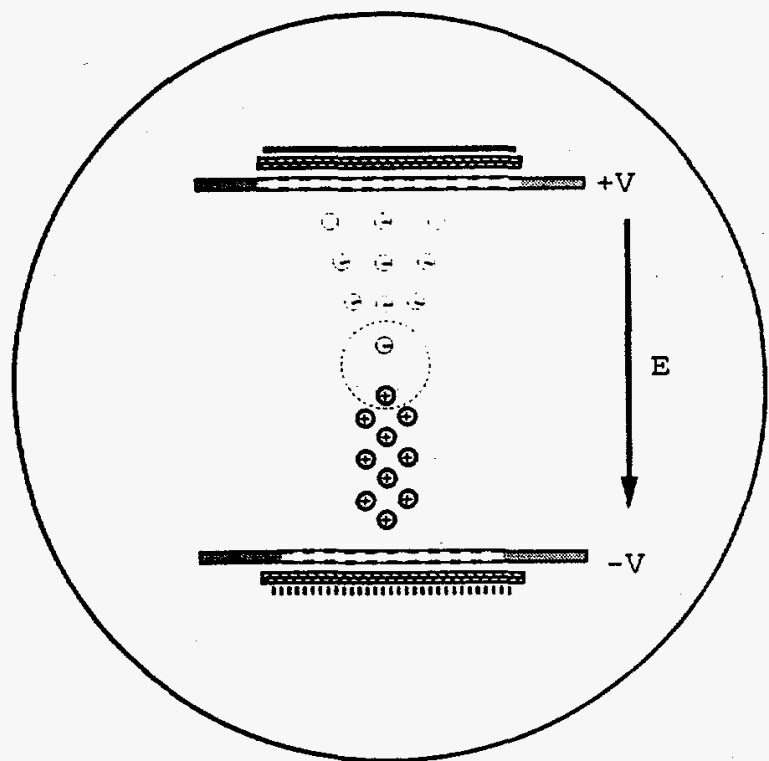

Fig. 3. Principles of Detector. Residual gas ion and electron pairs formed in collisions with beam particles are swept vertically in the direction of an applied electric field. Microchannel electron multipliers amplify the signals and provide timing and position signals for the postive ion hits and timing only for electron hits.

$$
T=\sqrt{\frac{2 M D z}{z \Delta V}-\sqrt{\frac{2 m D(D-z)}{e \Delta V}}}
$$

The variables in use here are the same as in Eq. (1) with the additional variable $m$, the electron mass. Typical electron drift times are around $1 \mathrm{~ns}$ whereas the ion drift times are hundreds of nanoseconds. The difference in drift times depends on the mass of the ion and the position at which the ion-electron pair was generated.

The anticipated sampling rate can be estimated using calculated energy loss due to gas ionization by the particle passing the sensitive region of the detector. The energy loss is then divided by the energy required to produce an electron ion pair from a gas molecule. The result is a small number, interpreted as an ionization probability that is proportional to the sampling probability (Microchannel efficiency may also be a factor). The numbers in Table 1 were calculated for $6 \mathrm{MeV} \alpha$ particles assuming a residual gas pressure of $5 \times 10^{-6}$ torr and a sampling region of $4 \mathrm{~cm}$. In Table $1, \Delta(\mathrm{eV})$ is the energy loss calculated for an areal thickness represented by $T\left(\mathrm{ng} / \mathrm{cm}^{2}\right)$. The ionization potentials for all these molecules are very similar, but the energy spectrum of electrons emitted following the interaction depends on the process, e.g. impact parameter. The average energy imparted to the ion and electron following an ionizing interaction is nonzero and depends also on the size, charge and mass of the molecule. These numbers are presented in the fifth column Table 1 and are taken from Ref. (9) and (10).

\section{CONSTRUCTION DETAILS AND DETECTOR TESTS}

The detector construction can be seen in the front view shown in Fig. 4. The electrodes are suspended from one plate using ceramic insulators and all electrical connections are fed through this plate. The sensitive volume is surrounded by two gridded electrodes which allow for $94 \%$ transmission of electrons and ions accelerated toward them. On both sides of the volume separating these electrodes we have placed insulating plates lined with field grading strips. The dimensions of the detector assembly are shown for reference (approximately $5 " \times 5$ " $\times 2.5$ "). Note the region spanned by the projection of the microchannel detectors is about half that size resulting in a sensitive volume of $2 " \times 2 " \times 2.5^{\prime \prime}$.

Signal amplification was achieved with dual, $40-\mathrm{mm}$ diameter, microchannel plate assemblies (8). The microchannel detector's anode plate was substituted by a circuit board with 40 conducting strips $(1 \mathrm{~mm}$ pitch, $0.6 \mathrm{~mm}$ wide) connected to delay line taps providing $2 \mathrm{~ns}$ delay per tap. The electrons exiting the microchannel detector assembly $\left(10^{7}\right.$ amplification) hit the anode strips. The signals are read from both sides of the delay line and the delay is decoded into position information. The prompt signal signifying the arrival of the ions was extracted by capacitive coupling to the one of the electrodes used to bias the microchannel detectors. In this way we were able to obtain both the time and the position of the positive ions' arrival. 


\section{Position resolution}

Horizontal position was obtained by recording the time difference between the signals arriving at both ends of the delay line connected to the anode strips. Vertical position signals were obtained by recording the ion drift time as registered with a start signal from the electron arrival time.

As noted in the previous section the drift time depends on the vertical position and mass of the ion. In order to better understand and calibrate the spectrum two separate runs were taken. In both cases the system. was first evacuated to $5 \times 10^{-7}$ torr and then a controlled leak was introduced bringing the pressure up to $8 \times 10^{-6}$ torr. In one case $\mathrm{N}_{2}$ gas was introduced, and the spectrum shows three prominent peaks corresponding to $\mathrm{N}_{2}^{+}, \mathrm{N}^{+}$and $\mathrm{N}^{++}$. In the other case water vapor was introduced and the spectrum shows two prominent peaks attributed to $\mathrm{H}^{+}$and $\mathrm{H}_{2} \mathrm{O}^{+}$. The spectra of vertical drift times gated by one of the solid state detectors are shown in Fig. 5. Although some "random" background still persist the data is clustered into discrete groups corresponding to the different ion species that were ionized and accelerated to the microchannel detectors for detection. The ion velocity dependence on $\sqrt{m}(\mathrm{~m}=$ ion mass) is shown in the calibration curve of Fig. 6.

The question is now whether with all the different mass groups arriving at different time intervals can one still get some useful position information out of the ion drift time. By creating a controlled environment in which the system is pumped but by introducing of a $\mathrm{N}_{2}$ gas leak we can have vertical position definition.
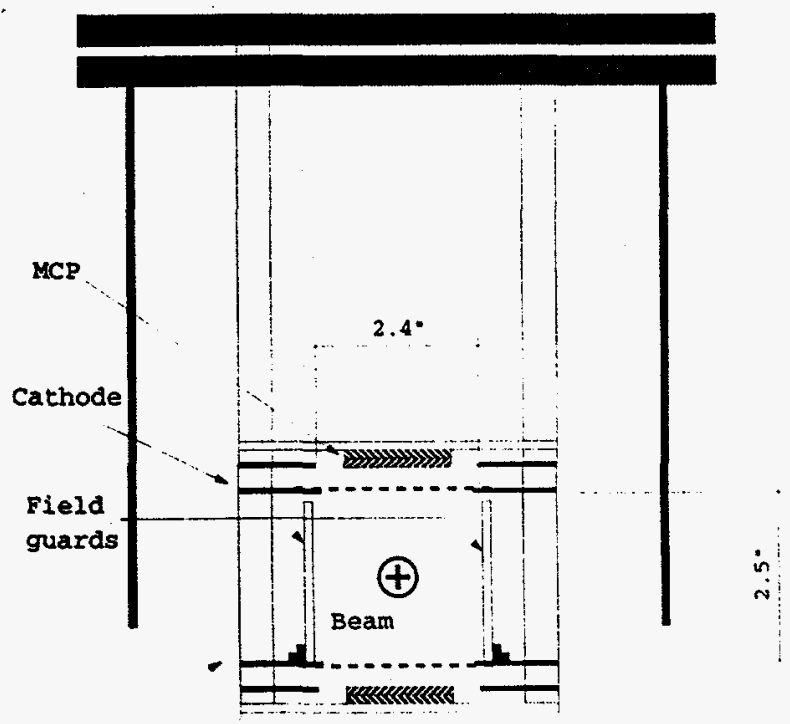

Anode

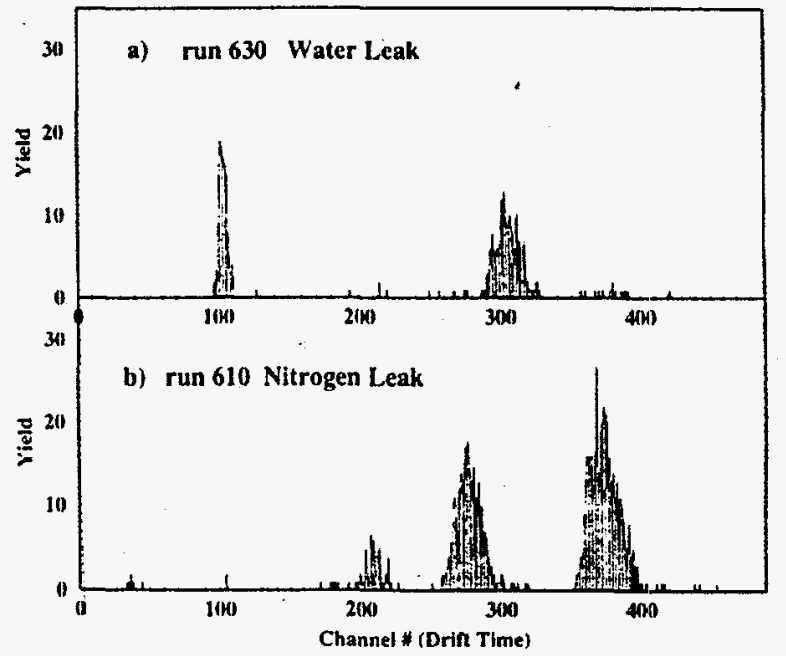

Fig. 5. Drift times of different ions produced in the residual gas doped with (a) a $\mathrm{H}_{2} \mathrm{O}$ leak and (b) a $\mathrm{N}_{2}$ leak.

\section{DETECTOR PERFORMANCE}

\section{Detection (ionization) efficiency}

Given the fact that there is always some background which cannot be eliminated, the sampling efficiency of the device is a predictor of its sensitivity at low beam intensities. The calculations which were presented in Table $l$ can serve as a guide. Efficiency measurements were done at three different pressures and demonstrate that the sampling rate is proportional to the ambient pressure. This proportionality is displayed over three orders of magnitude in the double logarithmic plot shown in Fig. 7 which displays measured sampling efficiency as a function of ambient pressure.

The beam profile monitor has been tested with stable beams and used in conjunction with a Coulomb excitation

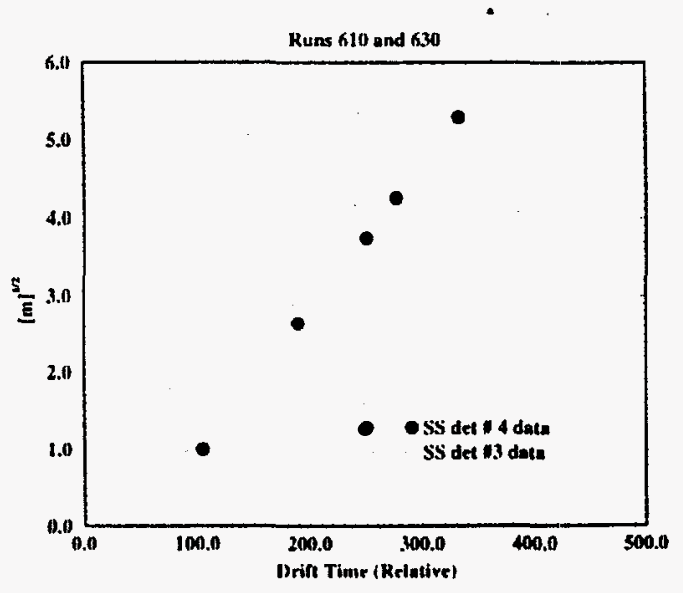

Fig. 6. $\wedge$ calibration curve showing drift time .vs. the square root of the mass of the aceelerated ion. The ions identified were H. N(doubly charged). N(singly charged). $\mathrm{H}_{2} \mathrm{O}$ and $\mathrm{N}_{2}$. 


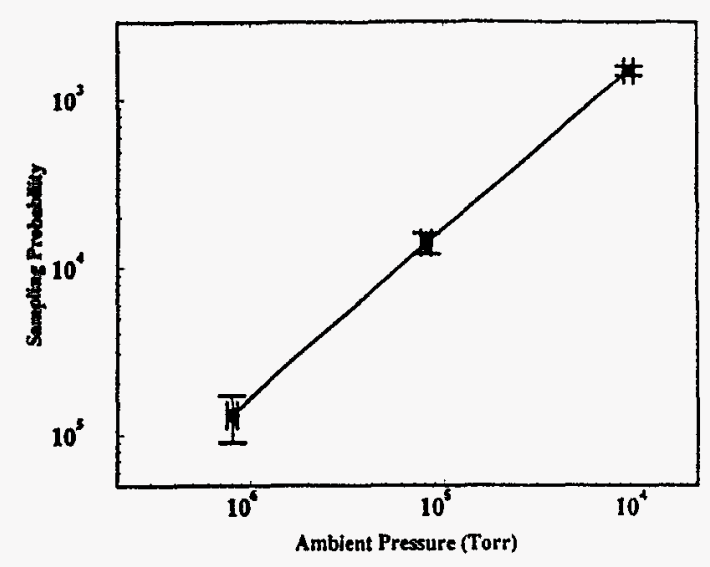

Fig. 7. Sampling rates as a function of ambient pressure.

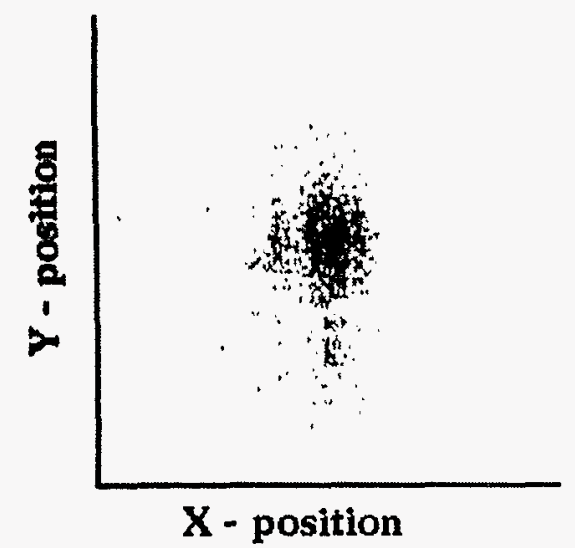

Fig. 8. Two-dimensional beam profile obtained with a beam of $10^{5}$ As ions per second.

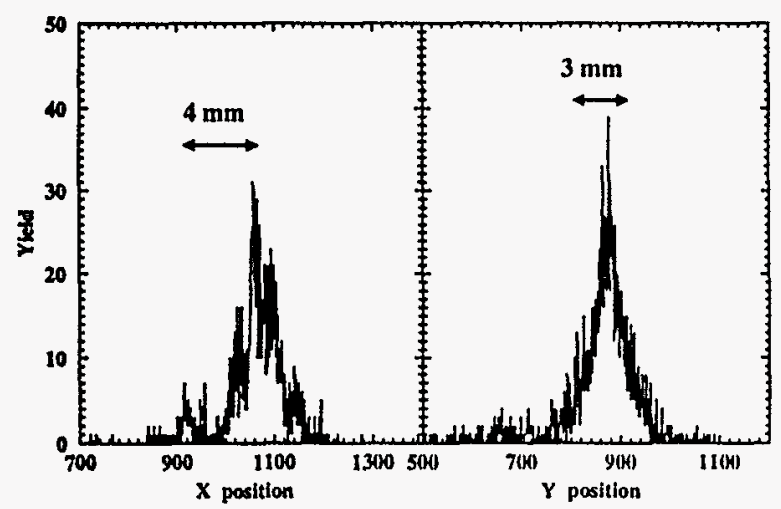

Fig. 9. Horizontal and vertical projection of beam spot obtained with a beam of $10^{569}$ As ions per second.

experiment done with ${ }^{69}$ As beams at the HRIBF facility.

Figure 8 shows a two dimensional plot of the beam profile obtained with ${ }^{69}$ As beam at intensitics near $10^{5}$ particles per second. The data shown was accumulated in less then one minute. The $\mathrm{Y}$ position was derived from the $\mathrm{N}_{2}$ drift time, and a biased TAC was used to amplify the region around that peak. As can be seen from the two orthogonal projections of the beam profile in Fig. 9, the resolutions in $X$ and $Y$ are comparable and the beam spot in this experiment was about $2-3 \mathrm{~mm}$ in diameter.

\section{CONCLUSION}

The horizontal position resolution obtained in our tests can be easily improved. By using more strips with, a smaller pitch one can achieve the expected resolving power of $0.3 \mathrm{~mm}$ realized in Ref. (5). We plan to use this device with extracted beam and may opt to use two-dimensional position sensing, such as the resistive layer used in Ref. (4).

\section{ACKNOWLEDGEMENT}

Oak Ridge National Laboratory is managed by Lockheed Martin Energy Research Corp. for the U.S. Department of Energy under contract number DE-AC05-96OR22464.

\section{REFERENCES}

I. T. Hardek, W. Kells and H. Lai, IEEE Trans. Nucl. Sci. NS-28 (1981) 2386.

2. J. Krider NIM A 278 (1989) 660.

3. A. N. Stillman, R. Thern, and R. L. Witkover, Rev. Sci. Instrum. 63 (6), 1992.

4. T. Quinteros, R. Schuch, M. Pajek, P. Sigray, H. Cerequist, H. Danared, L. Bagge, A. Filevich, J. Jeansson, A. Kallberg, and A. Paal, NIM A 333 (1993) 288.

5. B. Hochadel, F. Albrecht, M. Grieser, D. Habs, D. Schwalm, E. Szmola, and A. Wolf, NIM A 343 (1994) 401.

6. W. S. Graves, V. Bharadwaj, D. McGinnis, NIM A 364 (1995) 15.

7. W. S. Graves, NIM A 394 (1995) 19.

8. D. Shapira, T. A. Lewis, and J. L. Mosher, NIM A 400 (1997) 185.

9. Dual $40 \mathrm{~mm}$ MCP Model $640 \mathrm{C}$ COMSTOCK Inc., 1006 Alvin Weinberg Drive, Oak Ridge, TN. 37830, USA.

10. R. Meinke, W. Nexsen, E. Tsyganov, and A. Zinchebko, SSC Report.

I1. F. Sauli, CERN 77-09, 1975. 